\title{
Automorphisms of Rational Maps
}

\section{Citation}

McMullen, Curtis T. 1988. Automorphisms of rational maps. In Holomorphic Functions and Moduli I, ed. D. Drasin, C. J. Earle, F. W. Gehring, I. Kra, and A. Marden, Vol. 10, Mathematical Sciences Research Institute Publications, 31-60. New York: Springer.

\section{Permanent link}

http://nrs.harvard.edu/urn-3:HUL.InstRepos:9925395

\section{Terms of Use}

This article was downloaded from Harvard University's DASH repository, and is made available under the terms and conditions applicable to Other Posted Material, as set forth at http:// nrs.harvard.edu/urn-3:HUL.InstRepos:dash.current.terms-of-use\#LAA

\section{Share Your Story}

The Harvard community has made this article openly available.

Please share how this access benefits you. Submit a story.

\section{Accessibility}




\title{
Automorphisms of Rational Maps
}

\author{
Curt McMullen
}

\section{Introduction.}

Let $f(z)$ be a rational map, Aut(f) the finite group of Möbius transformations commuting with $f$. We study the question: when can two kinds of more flexible automorphisms of the dynamics of $\mathrm{f}$ be realized in $\operatorname{Aut}(\mathrm{g})$ for some deformation $g$ of $\mathrm{f}$ ?

First let Mod(f) denote the group of isotopy classes of quasiconformal maps commuting with $\mathrm{f}$. This group acts naturally on the Teichmüller space of f. It is often infinite and quite complicated. Nevertheless, if $\mathrm{G}$ is any finite subgroup of $\operatorname{Mod}(\mathrm{f})$, it can be realized as $\operatorname{Aut}(\mathrm{g})$ for some deformation $\mathrm{g}$ of $f(\S 2)$. (The proof uses Nielsen realization for Riemann surfaces.)

Now let $\operatorname{Mod}(\mathrm{J}, \mathrm{f})$ denote quasiconformal maps only required to commute with $\mathrm{f}$ on its Julia set $\mathrm{J}$. Elements of $\operatorname{Mod}(\mathrm{J}, \mathrm{f})$ arise naturally as monodromy in bifurcation-free families of rational maps. If the Julia set is connected and carries no invariant line field, $\operatorname{Mod}(\mathrm{J}, \mathrm{f})$ is a finite group $(\S 3)$. The proof uses realization in reverse: we show $\operatorname{Mod}(\mathrm{J}, \mathrm{f})$ is isomorphic to $\operatorname{Aut}(\mathrm{g})$ for some $\mathrm{g}$.

In the analogy with Kleinian groups, Aut(f) plays the role of the isometry group of the quotient hyperbolic manifold, while $\operatorname{Mod}(\mathrm{f})$ and $\operatorname{Mod}(\mathrm{J}, \mathrm{f})$ both resemble the manifold's mapping class group. Finiteness of $\operatorname{Mod}(\mathrm{J}, \mathrm{f})$ might be construed as the analogue of Johansson's theorem in three dimensional topology (connectedness of $\mathrm{J}$ is equated with incompressible boundary $(\S 4)$ ).

Studying the realization of $\operatorname{Mod}(\mathrm{J}, \mathrm{f})$ sheds light on the structure of periodic components of $\mathrm{J}$. In fact if $K$ is a component of $J$ such that $f(K)=K$, then there is a rational map $g$ such that the action of $f$ on $\mathrm{K}$ is quasiconformally conjugate to the action of $\mathrm{g}$ on its Julia set $\mathrm{J}(\mathrm{g})$. The proof uses 'conformal surgery' and a classification of the dynamics on the ideal boundary of $\mathrm{K}(\S \S 5,6)$.

A perhaps unexpected subtlety is that $\mathrm{K}$ might not meet the boundary of any component of the domain of normality $\hat{\mathbf{C}}-\mathrm{J}$ (see Figure I.1 and $\S 7$ ).

It is conjectured that when $\mathrm{J}$ is connected and $\mathrm{f}$ is nonaffine, $\operatorname{Mod}(\mathrm{J}, \mathrm{f})$ is always a finite group. Using a uniqueness criterion of Strebel's we establish this conjecture in the case of 'exposed critical points' (§ 8.)

In a sequel [Mc2] these finiteness results are shown to limit the possible braids arising from the motion of the attractor, which in turn leads to a topological criterion for the failure of iterative algorithms.

\section{$\S$ 1. Conformal Automorphisms}

Let $f(z)$ be a rational map of degree 2 or more. We denote by Aut(f) the group of Möbius transformations such that $\mathrm{f}(\mathrm{Mz})=\mathrm{M}(\mathrm{fz})$ for all z. For each $\mathrm{n}, \mathrm{M}$ permutes the finitely many periodic points of order n; since these number more than 3 for $n$ large enough, Aut(f) is a finite group.

If $G$ is a subgroup of Aut(f), we can form a new rational map f/G. Namely we form the orbifold $\hat{\mathbf{C}} / \mathrm{G}$, whose underlying manifold is again a sphere. Then $\mathrm{f}$ determines an endomorphism $\mathrm{f} / \mathrm{G}$ of $\hat{\mathbf{C}} / \mathrm{G}$, which is certainly conformal away from a finite set of points, and hence is again a rational map (of the same degree as f). 


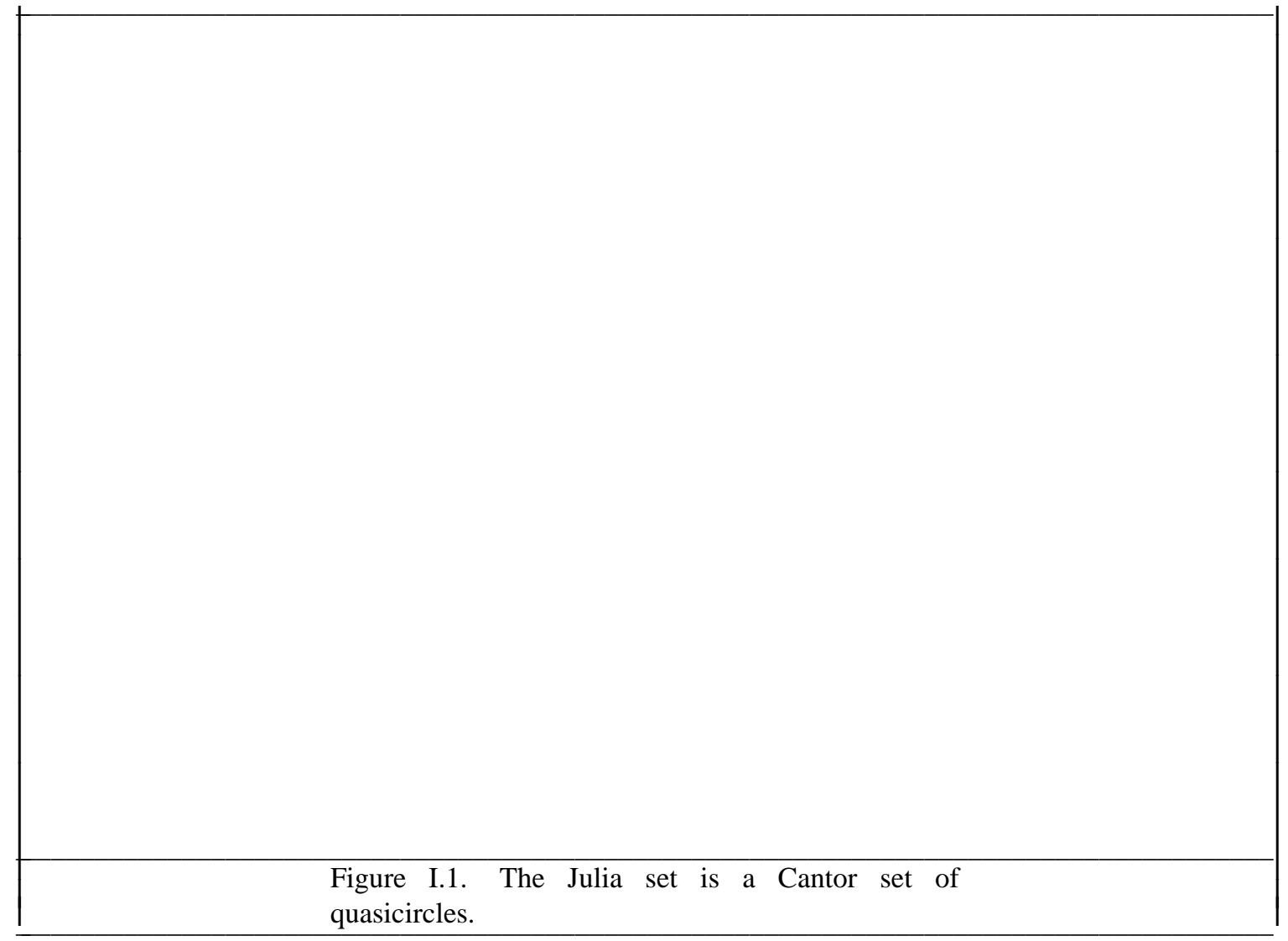

\section{Examples.}

(1) Let $\mathrm{f}(\mathrm{z})=\mathrm{z}^{\mathrm{n}+1}$; then Aut (f) is the dihedral group generated by $\mathrm{z} \rightarrow 1 / \mathrm{z}$ and $\mathrm{z} \rightarrow \omega \mathrm{z}$ where $\omega$ is a primitive nth root of unity. $\mathrm{T}(\mathrm{z})=\left(\mathrm{z}^{\mathrm{n}}+\mathrm{z}^{-\mathrm{n}}\right) / 2$ gives the orbifold covering $\hat{\boldsymbol{C}} \rightarrow \hat{\mathbb{C}} / \operatorname{Aut}(\mathrm{f})$, and $\mathrm{T}(\mathrm{f}(\mathrm{z}))$ $=\mathrm{g}(\mathrm{Tz})$, where $\mathrm{g}$ is the quotient rational map $\mathrm{f} / \operatorname{Aut}(\mathrm{f})$. If $\mathrm{z}=\mathrm{e}^{\mathrm{i} \theta / \mathrm{n}}$, then

$$
T(f(z))=\cos (n+1) \theta=g(\cos \theta),
$$

so $g(z)$ is the $(n+1)$ st Tchebycheff polynomial.

(2) The construction works for more general Riemann surfaces. Let $\mathrm{E}$ be a one dimensional complex torus (elliptic curve) and let $\mathrm{f}(\mathrm{z})=\mathrm{nz}$ in the group law on E. Then Aut(f) contains the group of order two $G$ generated by $z \rightarrow-z$, and $E / G \simeq \hat{\mathbb{C}}$ (the covering map is the Weierstrass p-function.) The map $\mathrm{g}=\mathrm{f} / \mathrm{G}$ is rational and satisfies $\mathrm{g}(\mathrm{p}(\mathrm{z}))=\mathrm{p}(\mathrm{nz})$.

Definition. A rational map is affine if it is obtained as the quotient of multiplication by an integer on a complex torus as in (2).

The Julia set of an affine map is the whole Riemann sphere. These are the only known maps which carry an invariant Beltrami differential supported on the Julia set (arising from the parallel line field on E). If they are the only such maps, then Axiom A dynamics is open and dense in the space of all rational functions ([Sul3], [Ma]). See [L] and [Mc1] for related discussions.

Much of the sequel will be devoted to realizing less rigid automorphisms of $f$ as elements of Aut(g) for some deformation $g$ of $\mathrm{f}$. 


\section{§ 2. Quasiconformal Automorphisms}

Definition. Let $\mathrm{QC}(\mathrm{f})$ denote the group of all quasiconformal maps $\phi: \hat{\mathbf{C}} \rightarrow \hat{\mathbf{C}}$, such that $\mathrm{f}(\phi(\mathrm{z}))=$ $\phi(f(z))$ for all z. The modular group of $\mathrm{f}($ denoted $\operatorname{Mod}(\mathrm{f}))$ is the group of isotopy classes of such $\phi$ : in other words, it is the quotient of QC(f) by the path component of the identity.

$\operatorname{Mod}(f)$ acts on Teich(f), the Teichmüller space of $f$, a contractible complex manifold which consists of pairs of rational functions and quasiconformal maps $\langle[\mathrm{g}],[\psi]\rangle$ such that $\psi$ establishes a quasiconformal conjugacy between $\mathrm{f}$ and $\mathrm{g}(\psi(\mathrm{g}(\mathrm{z}))=\mathrm{f}(\psi(\mathrm{z})))$. The brackets indicate that only the conformal conjugacy class of $g$ and the isotopy class of $\psi$ are remembered. The action is the obvious one: $[\phi]\langle[\mathrm{g}],[\psi]\rangle=\left\langle[\mathrm{g}],\left[\phi^{\circ} \psi\right]\right\rangle$. Any two choices for $\psi$ differ by an element of $\operatorname{Mod}(\mathrm{f})$, so the quotient of Teich(f) by this action amounts to forgetting $\psi$ altogether. The quotient $\mathbf{M}(\mathrm{f})$ is the moduli space of $\mathrm{f}$, consisting of conformal equivalence classes of rational maps quasiconformally conjugate to $f$. The action of $\operatorname{Mod}(f)$ is properly discontinuous, so $\mathbf{M}(\mathrm{f})$ is a complex orbifold [Sul3].

There is an inclusion $\operatorname{Aut}(\mathrm{f}) \subset \operatorname{Mod}(\mathrm{f})$; in fact $\operatorname{Aut}(\mathrm{f})$ is just the isotropy subgroup of $<[\mathrm{f}]$, [id] $>$.

Theorem 2.1. Let $\mathrm{G}$ be a finite subgroup of $\operatorname{Mod}(\mathrm{f})$. Then there is a rational map g, quasiconformally conjugate to $f$, such that $G$ is realized as a subgroup of $\operatorname{Aut}(\mathrm{g})$.

Proof. We will lift the isotopy classes represented by $\mathrm{G}$ to a finite group of quasiconformal homeomorphisms commuting with $\mathrm{f}$.

Let $\mathrm{E}$ denote the grand orbits of the periodic points and critical points of $\mathrm{f}$. Any element of QC(f) must carry this countable dynamically-defined set into itself, and hence its values on $\mathrm{E}$ are constant during isotopy. Thus each isotopy class in $\mathrm{G}$ already determines a well-defined homeomorphism on E.

By the classification of stable regions [Sul3], the complement of $\mathrm{E}^{-}$can be decomposed into two totally invariant open sets, the first of which consists of punctured disks and annuli coming from Herman rings, Siegel disks and superattracting basins. Any $[\phi]$ in $\mathrm{G}$ commutes with a conformal $S^{1}$ action on these regions, and hence respects the rigid circular structure on their ideal boundaries. A canonical lift is determined by isotoping $\phi$ to be conformal on the punctured disks and an affine stretch on annuli.

The second open set collapses to a (possibly disconnected) hyperbolic Riemann surface of finite volume when divided by the grand orbit equivalence relation. $G$ determines a finite group of isotopy classes on the quotient surface, which by Nielsen realization (Zieschang [Z, 54.3] and Kerckhoff [Ker]) can be lifted to a finite group of quasiconformal surface homeomorphisms. When pulled back to the domain of normality of $f$, this lift combines with the preceding choices to determines a lift of $G$ to a finite subgroup $G$ ' of quasiconformal maps commuting with $f$ on the whole Riemann sphere.

Since $G^{\prime}$ is finite, its elements are uniformly quasiconformal, and therefore some measurable complex structure is preserved simultaneously by $\mathrm{G}^{\prime}$ and $\mathrm{f}$ (cf. [Sul1, Theorem 9]). Using the measurable Riemann mapping theorem $[\mathrm{AB}]$, we obtain a quasiconformal map conjugating $\mathrm{f}$ to a new rational function $\mathrm{g}$ and taking $\mathrm{G}$ ' to a finite group of Möbius transformations commuting with $\mathrm{g}$, as claimed.

Definition. A rational map $\mathrm{f}(\mathrm{z})$ is critically finite if its critical points are preperioidic $\left(\mathrm{f}^{\mathrm{n}}(\mathrm{c})=\mathrm{f}^{\mathrm{m}}(\mathrm{c})\right.$ for some $n \neq m$.)

Theorem 2.2. Let $f(z)$ be a critically finite rational map. If $f$ is not affine, then $\operatorname{Aut}(f) \simeq \operatorname{Mod}(f)$. In other words any quasiconformal automorphism is isotopic to a conformal automorphism.

This result is implicit in a theorem of Thurston (see [DH2]); it is worked out explicitly in [Mc1,8.1]. In particular, the modular group is finite. 
Remark. The Julia set of a critically finite map is connected. To see this, note that under iteration, every critical point outside the Julia set eventually lands on a periodic critical point. Thus the immediate basin of attraction of a periodic critical point contains no others, so it is a disk. Furthermore, each component of the complement of the Julia set eventually lands on a superattracting basin, so it admits a proper map to a disk branched over only one point (the superattracting cycle). Hence every complementary component is a disk, and the Julia set is connected.

In the following section we will see that connectedness of the Julia set already has strong consequences for automorphisms of the dynamics.

\section{Examples.}

(1) If $\mathrm{f}$ is affine, $\operatorname{Mod}(\mathrm{f}) \simeq \mathrm{PSL}_{2} \mathbf{Z}$. (As observed by Herman $[\mathrm{H}]$, the linear action of $\mathrm{SL}_{2} \mathbf{Z}$ on the torus descends to automorphisms of $\mathrm{f}$ ).

(2) Let $f(z)=\lambda z+z^{2}$ for $0<|\lambda|<1$. The Julia set of $f$ is a quasicircle, and the quotient Riemann surface is the union of a foliated disk and a once-punctured torus (corresponding to the basins of $\infty$ and 0 respectively.) Mod(f) is an infinite cyclic group generated by a Dehn twist about the unique simple closed curve on the torus which lifts to a circle enclosing 0 . In fact the parameter $\lambda$ establishes an isomorphism of the moduli space $\mathbf{M}(\mathrm{f})$ with the punctured unit disk.

Problem. For $\mathrm{f}(\mathrm{z})$ a generic perturbation of $\mathrm{z} \rightarrow \mathrm{z}^{\mathrm{n}}, \mathrm{n}>2$, the quotient Riemann surface is a pair of (n-1)-fold punctured tori (here generic means all $2 \mathrm{n}-2$ critical points are simple and have distinct grand orbits). Mod(f) is a subgroup of the mapping class group of this Riemann surface; what subgroup is it?

\section{$\S$ 3. Stable Conjugacy}

Definitions. Let $f(z), g(z)$ be two rational maps, not necessarily of the same degree. Let $F, G$ be closed subsets of the Riemann sphere $\hat{\mathbb{C}}$ such that $\mathrm{f}(\mathrm{F})=\mathrm{F}, \mathrm{g}(\mathrm{G})=\mathrm{G}$. A map $\phi: \hat{\mathbb{C}} \rightarrow \hat{\boldsymbol{C}}$ determines a stable conjugacy $(\mathrm{F}, \mathrm{f}) \sim(\mathrm{G}, \mathrm{g})$ if

(1) $\phi$ is quasiconformal on $\hat{\mathbf{C}}$;

(2) $\phi(F)=G$; and

(3) $\phi^{\circ} f(z)=g^{\circ} \phi(z)$ for all $z$ in $F$.

By definition, two maps $\phi$ and $\psi$ to determine the same stable conjugacy if they are isotopic through maps enjoying (1)-(3).

The stable self-conjugacies $(\mathrm{F}, \mathrm{f}) \sim(\mathrm{F}, \mathrm{f})$ form a group, the modular group of $f$ on $F$, which we denote by $\operatorname{Mod}(F, f)$. Of particular importance is the case $F=J(f)$, the Julia set of $f$. A stable conjugacy $\mathrm{f} \sim \mathrm{g}$ will mean a conjugacy on their respective Julia sets. If $\mathrm{f} \sim \mathrm{g}$ then $\operatorname{Mod}(\mathrm{J}(\mathrm{f}), \mathrm{f}) \simeq \operatorname{Mod}(\mathrm{J}(\mathrm{g}), \mathrm{g})$.

A family of rational maps $\mathrm{f}_{\lambda}(\mathrm{z})$ is holomorphic if the coefficients of $\mathrm{f}$ are holomorphic functions of the parameter $\lambda$. Such families obey a strong dichotomy: either they display a rich cascade of bifurcations, or their members look rather like one another. To formulate the latter property, we say a holomorphic family is stable if there is a uniform upper bound on the periods of attracting cycles for maps occurring in the family. Members of a stable family need not be quasiconformally conjugate on the whole Riemann sphere, but they are stably conjugate on their Julia sets [MSS]. This will be made precise below.

Let $\mathrm{X}$ be a complex manifold parameterizing a stable holomorphic family of rational maps, including points corresponding to maps $\mathrm{f}(\mathrm{z})$ and $\mathrm{g}(\mathrm{z})$. Let $\mathrm{A}(\mathrm{f})$ denote the set of preperiodic points other than those in the Julia set.

A(f) is just the union of the grand orbits of the attracting and superattracting points and the centers of Siegel disks. 
Proposition 3.1. The homotopy class of a path $\gamma$ connecting $\mathrm{f}$ to $\mathrm{g}$ in $\mathrm{X}$ determines a stable conjugacy

$$
(\mathrm{J}(\mathrm{f}) \cup \mathrm{A}(\mathrm{f}), \mathrm{f}) \sim(\mathrm{J}(\mathrm{g}) \cup \mathrm{A}(\mathrm{g}), \mathrm{g}) .
$$

Proof. By [MSS], in a stable holomorphic family of rational maps, the periodic points move without collision; the motion extends to the closure of the preperiodic points $(=\mathrm{J} \cup \mathrm{A})$ and commutes with the dynamics. If we follow the motion along $\gamma$ from $f$ to $g$ we obtain a conjugacy $\phi$ mapping $J(f) \cup A(f)$ to $\mathrm{J}(\mathrm{g}) \cup \mathrm{A}(\mathrm{g})$ and depending only on the homotopy class of $\gamma$.

The holomorphic motion of J $\cup A$ can be locally extended to a holomorphic motion of the whole Riemann sphere [ST], [BR], which is then quasiconformal as a function of $\mathrm{z}$. The isotopy class of the extension is unique (this is true for any extension of an isotopy of a set to an ambient isotopy.)

Breaking the path $\gamma$ into small segments, we see the composition of the local extensions defines a map $\phi$ satisfying conditions (1-4). Since the isotopy class of extension is locally well-defined, it does not change as we vary $\gamma$ by homotopy; consequently the homotopy class of $\gamma$ determines a stable conjugacy.

Corollary 3.2. Holomorphic motion around loops in $X$ based at $f$ determines a monodromy map

$$
\pi_{1}(\mathrm{X}, \mathrm{f}) \rightarrow \operatorname{Mod}(\mathrm{J}(\mathrm{f}) \cup \mathrm{A}(\mathrm{f}), \mathrm{f}) .
$$

The study of this map will be central to the discussion in [Mc2].

Definition. A line field on the Julia set is a measurable Beltrami coefficient $\mu(\mathrm{z}) \mathrm{dz} / \mathrm{dz}$ supported on a positive area subset of $\mathrm{J}$, such that on its support, $|\mu|=$ const. $>0$. The line field is invariant if $\mathrm{f}^{*} \mu=\mu$. The invariant Beltrami differentials supported on the Julia set form a finite dimensional vector space with a basis of invariant line fields [Sul3].

In all known examples (other than $\mathrm{f}$ affine), the Julia set carries no invariant line field at all.

Theorem 3.3. Let $f(z)$ be a rational map with connected Julia set $J$ carrying no invariant line field. Then there is a rational map g, stably conjugate to $\mathrm{f}$, such that $\operatorname{Mod}(\mathrm{J}, \mathrm{f})$ is realized as $\operatorname{Aut}(\mathrm{g})$. In particular $\operatorname{Mod}(\mathrm{J}, \mathrm{f})$ is a finite group.

Conjecture. For any nonaffine rational map with connected Julia set, $\operatorname{Mod}(\mathrm{J}, \mathrm{f})$ is a finite group.

In $\S 8$ we will establish the conjecture in the case of 'exposed critical points'.

The map $g$ above is obtained by surgically altering $f$ off of $J$ so its quotient Riemann surface has no moduli. The process can even be applied to a component of $\mathrm{J}$, yielding a more general result:

Theorem 3.4. Let $K$ be a component of the Julia set of $f$ (other than a single point) such that $f(K)=K$. Then:

(a) There exists a rational map $\mathrm{g}$ with Julia set $\mathrm{J}(\mathrm{g})$ and a stable equivalence $\phi:(\mathrm{K}, \mathrm{f}) \sim$ $(\mathrm{J}(\mathrm{g}), \mathrm{g})$. The map $\phi$ determines an isomorphism $\operatorname{Mod}(\mathrm{K}, \mathrm{f}) \simeq \operatorname{Mod}(\mathrm{J}(\mathrm{g}), \mathrm{g})$.

(b) If $\mathrm{K}$ above carries no invariant line field, then $\mathrm{g}$ can be chosen so $\operatorname{Mod}(\mathrm{J}(\mathrm{g}), \mathrm{g}) \simeq \operatorname{Aut}(\mathrm{g})$; in particular $\operatorname{Mod}(K, f)$ is a finite group.

The proof of this theorem occupies $\S \S 5$ and 6 .

Corollary 3.5. The Julia set of a rational map has at most countably many preperiodic components. The set of (pre)periodic points is dense in each (pre)periodic component. 
Proof. The periodic points of $\mathrm{g}$ are dense in $\mathrm{J}(\mathrm{g})$.

If we start with an expanding map, the g constructed in Theorem 3.3 will turn out to be critically finite, which together with the remark following Theorem 2.2 establishes:

Corollary 3.6. An expanding rational map is stably conjugate to a critically finite map if and only if its Julia set is connected.

\section{Examples.}

(1) Let $f(z)=z^{n}+c$, for $c$ small. Then the Julia set of $f$ is a quasicircle, $f$ maps $J$ to itself by a degree $\mathrm{n}$ covering, and $\operatorname{Mod}(\mathrm{J}, \mathrm{f})$ is the dihedral group of symmetries of the (n-1) - gon obtained by marking the (n-1) fixed points of $\mathrm{f}$ in $\mathrm{J}$. This group is realized as the group of automorphisms of $\mathrm{g}(\mathrm{z})$ $=\mathrm{z}^{\mathrm{n}}$, which is stably conjugate to $\mathrm{f}$. Notice that $\mathrm{g}$ does not lie in the Teichmüller space of $\mathrm{f}$; it is for this reason that we replace quasiconformal conjugacy with the weaker notion of stable conjugacy.

(2) To see the assumption of connected Julia set is needed, let $f(z)=z^{3}+\lambda z^{2}$ for $\lambda$ large. Then in $[\mathrm{Mc} 1]$ we show the group of homeomorphisms $\operatorname{Mod}(\mathrm{f}) \mathrm{lJ}_{\mathrm{J}}$ (obtained by restricting each $[\phi]$ to its values on the Julia set) is infinite. Using the natural map $\operatorname{Mod}(f) \rightarrow \operatorname{Mod}(J, f)$ it follows that $\operatorname{Mod}(J, f)$ is infinite as well. (In general this map is neither an injection nor a surjection, but it respects the values of $[\phi]$ on the Julia set.) Of course the Julia set for this map is not connected; it is a mixture of a Cantor set and a countable number of quasicircles.

(3) A simpler example is the map $\mathrm{f}(\mathrm{z})=\mathrm{z}^{3}+\lambda$ for $\lambda$ large. The Julia set is a Cantor set and the action of $\mathrm{f}$ on $\mathrm{J}$ is topologically conjugate to $\left(\Sigma_{3}, \sigma\right)$, the one-sided shift on three symbols. $\left.\operatorname{Mod}(\mathrm{J}, \mathrm{f})\right|_{\mathrm{J}}$ is the full group of topological automorphisms of the three shift.

Remark. When $\mathrm{J}$ is connected, one can construct a contractible complex manifold Stab(f) which parameterizes all maps stably conjugate to $\mathrm{f}$. $\operatorname{Stab}(\mathrm{f})$ consists of pairs $\langle[\mathrm{g}],[\phi]\rangle$, where $\phi$ gives a stable conjugacy $\mathrm{f} \sim \mathrm{g}$. $\operatorname{Mod}(\mathrm{J}, \mathrm{f})$ acts on this space, and the quotient furnishes a partial compactification of the moduli space of $\mathrm{f}$. Thus every element of $\operatorname{Mod}(\mathrm{J}, \mathrm{f})$ arises from monodromy in this universal family. One can think of Theorem 3.3 is providing a simultaneous fixed point for all elements of the modular group.

On the other hand, when $\mathrm{J}$ is not connected $\operatorname{Mod}(\mathrm{J}, \mathrm{f})$ is always too big: there exists elements which do not arise as the monodromy in any stable family. (There are too many choices for the isotopy class of $\phi$ on the complement of J.) Nevertheless it is a technically convenient group to work with.

Problem. Construct the stable deformation space of a rational map with possibly disconnected Julia set. Identify its modular group intrinsically in terms of $\mathrm{f}$.

\section{§ 4. Comparison with Hyperbolic Three Manifolds}

In [Sul2] a striking dictionary is established between the dynamics of rational maps and of Kleinian groups. In this section we attempt a translation of some of the preceding results. (Some contrasts are discussed in $§ 7$.)

Let $\Gamma$ be a finitely generated Kleinian group (discrete subgroup of $\mathrm{PSL}_{2} \mathbf{C}$ ) with limit set $\Lambda$, domain of discontinuity $\Omega$ and quotient three manifold $\mathrm{M}^{3}=\left(\mathbf{H}^{3} \cup \Omega\right) \Gamma$. For simplicity we assume $\mathrm{M}^{3}$ is convex cocompact, i.e. geometrically finite without cusps.

The Teichmüller space of deformations of $\mathrm{M}^{3}$ can be described as the set of pairs $\left\langle\left[\Gamma^{\prime}\right],[\phi]\right\rangle$, where $\phi$ is a quasiconformal map conjugating $\Gamma$ to another Kleinian group $\Gamma^{\prime}$, and the brackets indicate the pair $\Gamma^{\prime}, \phi$ is determined only up to inner automorphism, conformal conjugacy and isotopy. Then Teich $\left(\mathrm{M}^{3}\right)$ is a contractible complex manifold, which can be identified with the Teichmüller space of the (possibly disconnected) Riemann surface $\Omega \Gamma$. 
Let $\operatorname{Mod}\left(\mathrm{M}^{3}\right)$ denote the group of quasiconformal maps conjugating $\Gamma$ to itself, modulo isotopy and inner automorphism. Then $\operatorname{Mod}\left(\mathrm{M}^{3}\right)$ is isomorphic to the mapping class group of $\mathrm{M}^{3}$. (Here it is essential that for the mapping class group of $\mathrm{M}^{3}$ we take autohomeomorphisms modulo isotopy.) The quotient of Teich $\left(\mathrm{M}^{3}\right)$ by $\operatorname{Mod}\left(\mathrm{M}^{3}\right)$ is the moduli space of complete hyperbolic structures on $\mathrm{M}^{3}$. (Our definitions differ slightly from those of [Kra], because we wish to identify $\operatorname{Mod}\left(\mathrm{M}^{3}\right)$ with the mapping class group and we wish to have Teich $\left(\mathrm{M}^{3}\right)$ contractible.)

Then Theorem 2.1 above has a precise parallel (which seems to be well known):

Proposition 4.1. If $\mathrm{G}$ is a finite subgroup of the mapping class group of $\mathrm{M}^{3}$, then there is a quasiisometry $\phi$ mapping $\mathrm{M}^{3}$ to another complete hyperbolic manifold $\mathrm{N}^{3}$, on which the group $\mathrm{G}$ is realized as isometries.

A proof can be given along the lines of Theorem 2.1, using e.g. Thurston [Th, Chapter 11] or Douady-Earle [DE] to obtain the quasi-isometry statement.

It is interesting to speculate on the connection between our finiteness result for $\operatorname{Mod}(J, f)$ when $J$ is connected and known results in the topology of three manifolds. By Dehn's lemma and the loop theorem, the limit set $\Lambda$ is connected if and only if $\mathrm{M}^{3}$ has incompressible boundary. Thus Theorems 3.3 and 3.4 above are reminiscent of the following result of Johansson $[\mathrm{J}]$ :

Theorem 4.2. If $\partial \mathrm{M}^{3}$ is incompressible, and every essential annulus is boundary parallel, then the mapping class group of $\mathrm{M}^{3}$ is finite.

Moreoever Thurston proved $\mathrm{AH}(\mathrm{M})$ is compact for such manifolds (see [MB]; $\mathrm{AH}(\mathrm{M})$ is the space of discrete faithful representations of $\Gamma$ in the algebraic topology). One can recognize these manifolds as follows: not only is the limit set connected, but no group element has fixed points which are both on the boundary of two different components of $\Omega$. If 'fixed points' is replaced by 'periodic point', one has a reasonable generalization to rational functions. Comparing with Thurston's theorem, we propose the following:

Problem. If $\mathrm{f}(\mathrm{z})$ is a rational function with connected Julia set and no periodic point on the boundary of two components of $\Omega$, is the closure of the moduli space $\mathbf{M}(\mathrm{f})$ compact in $\mathrm{Rat}_{\mathrm{K}} \mathrm{PSL}_{2} \mathbf{C}$ ?

(Here $\mathrm{Rat}_{\mathrm{k}}$ is the space of rational maps strictly of degree $\mathrm{k}$. The quotient space is noncompact because a map can go to infinity by dropping in degree.)

The example of a generic perturbation of $\mathrm{z}^{\mathrm{k}}$ shows that some hypothesis beside connectedness of $\mathbf{J}$ is necessary.

\section{$\S 5$. Conformal Surgery}

We begin the proof of 3.4 by describing the construction of isotopies and new rational functions. The gluing lemma (5.5) provides the surgical technique for producing stable conjugacies.

Definition. A disk will mean a simply connected open subset of $\hat{\mathbf{C}}$ whose complement contains at least 2 points. In this section $\mathrm{U}$ and $\mathrm{V}$ will always denote disks. The ideal boundary $\mathrm{I}(\mathrm{U})$ is the space of prime ends of $\mathrm{U}$. I $(\mathrm{U})$ can be identified with the usual boundary $\mathbf{S}^{1}$ of the unit disk $\Delta$ via a Riemann mapping. The identification is well-defined up to post-composition with a Möbius automorphisms of $\Delta$. Thus the ideal boundary has a natural projective (i.e. $\mathrm{PSL}_{2} \mathbf{R}$ ) structure. (For example, the cross-ratio of four prime ends makes sense.)

Proposition 5.1. Let $\phi: \hat{\mathbb{C}} \rightarrow \hat{\mathbb{C}}$ be a homeomorphism which is quasiconformal on a open region $\mathrm{R}$ and the identity on $\hat{\mathbf{C}}-\mathrm{R}$. Then $\phi$ is quasiconformal on the whole Riemann sphere.

See [DH1, Lemma 2] for a more general result. The above is also stated without proof in [Kra]. 


\section{Proposition 5.2.}

(a) Let $\phi: \mathrm{U}^{-} \rightarrow \overline{\mathrm{U}}$ be an orientation preserving homeomorphism which is the identity on the topological boundary of $\mathrm{U}$. Then $\phi$ is the identity on the ideal boundary.

(b) Let $\phi: U \rightarrow U$ is quasiconformal map of $U$ which is the identity on the ideal boundary of $U$. Then $\phi$ extends by the identity to a quasiconformal map of the whole Riemann sphere, which in particular is the identity on the topological boundary of $U$.

\section{Proof.}

(a) Let $\mathrm{C}_{\mathrm{n}}$ be a sequence of disjoint cross-cuts with distinct endpoints defining a prime end. Uniform continuity insures $\phi\left(\mathrm{C}_{\mathrm{n}}\right)$ defines another prime end, and by assumption the end points of each cross-cut are unchanged. Consider the quadrilateral $\mathrm{Q}$ in $\mathrm{U}$ bounded by $\mathrm{C}_{\mathrm{k}} \cup \mathrm{C}_{\mathrm{k}+1}$, for $\mathrm{k}$ large. Since $\phi$ is orientation preserving, both $\mathrm{Q}$ and its image lie in the same component of $\mathrm{U}-\mathrm{C}_{\mathrm{n}}$. It follows that $\phi\left(\mathrm{C}_{\mathrm{n}}\right)$ defines the same prime end as the sequence $C_{n}$, so $\phi$ is the identity on the ideal boundary of $U$. (This type of argument was shown to me independently by Hinkkanen and Douady.)

(b) A quasiconformal map which is the identity on the ideal boundary moves points only a bounded distance in the Poincare' metric on U. Since the Poincare' metric tends to $\infty$ relative to the spherical metric on $U, \phi$ extends continuously to the identity on $\partial \mathrm{U}$. Suppose we extend $\phi$ by the identity over the remainder of the sphere. The result is a homeomorphisms which is quasiconformal on $U$ and the identity on $\hat{\mathbf{C}}-\mathrm{U}$; so we may apply Proposition 5.1.

Proposition 5.3. Let $\phi, \psi: \overline{\mathrm{U}} \rightarrow \overline{\mathrm{V}}$ be continuous maps, K-quasiconformal on $\mathrm{U}$ and equal on $\partial \mathrm{U}$. Then there exists an isotopy $\phi_{\mathrm{t}}$ connecting $\phi$ to $\psi$ through $\mathrm{K}^{3}$-quasiconformal maps, all agreeing on $\partial \mathrm{U}$.

Proof. Let $\alpha=\psi^{-1} \circ \phi: U \rightarrow U$. Then (i) $\alpha$ is $\mathrm{K}^{2}$-quasiconformal and (ii) $\alpha$ equals the identity on the ideal boundary of $\mathrm{U}$. We can construct an isotopy $\alpha_{t}$ connecting $\alpha$ to the identity map through homeomorphisms enjoying these two properties. Just uniformize $U$ and bring the identity in radially from the boundary of the unit disk.

By 5.1 each $\alpha_{\mathrm{t}}$ extends to the identity on $\partial \mathrm{U}$. The desired isotopy is given by $\phi_{\mathrm{t}}=\psi^{\circ} \alpha_{\mathrm{t}}$.

Corollary 5.4. If $K$ is connected and periodic points of $f$ are dense in $K$, then any $[\phi]$ in $\operatorname{Mod}(K, f)$ is uniquely determined by its values on $\mathrm{K}$.

Remark. Density of periodic points implies the value of $[\phi]$ on $\mathrm{K}$ is well-defined, since any isotopic map must agree with $\phi$ on periodic points.

Proof. Suppose $[\phi]$ is the identity on K. Then $\phi$ is the identity on the ideal boundary of every disk in the complement of $\mathrm{K}$. By the above propositions, on each disk there is an isotopy connecting $\phi$ to the identity through quasiconformal maps which are the identity on $\mathrm{K}$. The dilatation of the intervening maps is bounded by $\mathrm{K}(\phi)^{3}$ independent of the disk. By 5.1 the time t maps in each disk piece together to form a quasiconformal map of the whole Riemann sphere. Thus $[\phi]=[i d]$ in $\operatorname{Mod}(\mathrm{K}, \mathrm{f})$.

We now turn to the Gluing Lemma, which allows one to redefine a rational map $f(z)$ on a disk $U$ using a new map which is compatible on with $f$ on the ideal boundary.

Definitions. Let $K \subset \hat{\mathbf{C}}$ be an infinite continuum (i.e. a continuum which is neither empty nor a single point). Then every component of $\hat{\mathbf{C}}-\mathrm{K}$ is a disk and we define $\mathrm{I}(\mathrm{K})$, the ideal boundary of $K$, to be the union of the ideal boundaries of its complementary components.

Let $\mathrm{f}$ be a rational map such that $\mathrm{f}^{-1}(\mathrm{~K})$ consists of $\mathrm{K}$ itself and finitely many other disjoint components. (In applications, $K$ will be a component of the Julia set such that $f(K)=K$ ). 
Then the map f gives rise to a real-analytic map $\mathrm{f}: \mathrm{I}(\mathrm{K}) \rightarrow \mathrm{I}(\mathrm{K})$ as follows. Let $\mathrm{U}$ be a component of $\hat{\mathfrak{C}}-\mathrm{K}$, and let $\mathrm{U}^{\prime}$ denote the component of $\mathrm{U}-\mathrm{f}^{-1} \mathrm{~K}$ whose closure contains $\partial \mathrm{U}$. Then $\mathrm{f}$ carries $\mathrm{U}^{\prime}$ to $\mathrm{V}$ by a proper map, where $\mathrm{V}$ is another component of $\hat{\boldsymbol{C}}-\mathrm{K}$. On the uniformizations of $\mathrm{U}$ and $\mathrm{V}, \mathrm{f}$ carries a neighborhood of $\mathbf{S}^{1}$ to a neighborhood of $\mathbf{S}^{1}$. By Schwarz reflection, $f$ extends to a real-analytic map from $\mathrm{I}(\mathrm{U})$ to $\mathrm{I}(\mathrm{V})$.

Note that we cannot assume that $f(U)=V$ and in fact $f(U)$ may cover the whole Riemann sphere many times.

The immediate result of surgery will not be a rational map, but a more flexible object:

Definition. A quasirational function is a branched covering $\mathrm{f}: \hat{\mathbf{C}} \rightarrow \hat{\mathbf{C}}$ which is

(1) locally conjugate to a holomorphic map after quasiconformal change of coordinates in the domain and range; and

(2) whose iterates $\mathrm{f}^{\mathrm{n}}$ are $\mathrm{K}$-quasiconformal (away from branch points) for some $\mathrm{K}$ independent of $n$.

By [Sul1, Theorem 9], a function is quasirational if and only if it is quasiconformally conjugate to a rational function.

To allow us to carry out a succession of surgeries without constantly converting the results back to rational functions, we will state the gluing lemma for quasirational functions. Using Sullivan's theorem, it is easy to check that the map $f: I(K) \rightarrow I(K)$ makes sense when $f$ is only quasirational; however the map on ideal boundary is then only quasisymmetrically conjugate to a real-analytic map.

Proposition 5.5 (The Gluing Lemma). Let $\mathrm{f}(\mathrm{z})$ be a quasirational function, and let $\mathrm{K}$ be an infinite continuum such that $\mathrm{f}^{-1}(\mathrm{~K})$ consists of $\mathrm{K}$ itself and finitely many other disjoint components. Let B : $\Delta \rightarrow \Delta$ be a proper holomorphic map (a finite Blaschke product).

(a) Suppose $\mathrm{f}: \mathrm{I}(\mathrm{U}) \rightarrow \mathrm{I}(\mathrm{U})$ for some component $\mathrm{U}$ of $\hat{\mathfrak{C}}-\mathrm{K}$, and $\phi: \mathrm{U} \rightarrow \Delta$ is a quasiconformal mapping whose ideal boundary values $\mathrm{I}(\mathrm{U}) \rightarrow \mathbf{S}^{1}$ give a conjugacy between the action of $\mathrm{f}$ on the ideal boundary of $\mathrm{U}$ and that of $\mathrm{B}$ on $\mathbf{S}^{1}$. Then the function

$$
\begin{aligned}
g(z) & =f(z) & & \text { for } z \text { not in } U \\
& =\phi^{-1}{ }^{\circ} B^{\circ} \phi(z) & & \text { for } z \text { in } U
\end{aligned}
$$

is quasirational.

(b) Suppose $U$ and $V$ are components of $\hat{\mathbb{C}}-\mathrm{K}$ such that $\mathrm{f}: \mathrm{I}(\mathrm{U}) \rightarrow \mathrm{I}(\mathrm{V})$ and $\mathrm{f}^{\mathrm{n}} \mathrm{I}(\mathrm{U}) \cap \mathrm{I}(\mathrm{U})=\varnothing$ for all $\mathrm{n}>0$. If $\phi, \psi$ are quasiconformal maps of $\mathrm{U}$ and $\mathrm{V}$ to $\Delta$ such that $\psi^{\circ} \mathrm{f}=\mathrm{B}^{\circ} \phi$ on the ideal boundary of $\mathrm{U}$, then

$$
\begin{aligned}
g(z) & =f(z) & & \text { for } z \text { not in } U \\
& =\psi^{-1}{ }^{\circ} B^{\circ} \phi(z) & & \text { for } z \text { in } U
\end{aligned}
$$

is quasirational.

Notation. For part (a) we will write $g=f \cup_{\phi} B$, for part (b) $g=f \cup_{(\phi, \psi)} B$.

Proof. In either case, consider $\mathrm{f}^{-1} \circ \mathrm{g}(\mathrm{z})$ locally near $\partial \mathrm{U}$. This map is the identity outside of $\mathrm{U}$, quasiconformal on $\mathrm{U}$ and the identity on the ideal boundary of $\mathrm{U}$. Therefore it is locally quasiconformal. Composing with $\mathrm{f}$, we conclude $\mathrm{g}$ is locally quasiconformal away from branch points. Moreover the dilation of $g$ is bounded by the supremum of $K(f)$ and $K(\phi)^{2}$.

In case (a), $\mathrm{g}^{\mathrm{n}}=\mathrm{f}^{\mathrm{n}} \cup_{\phi} \mathrm{B}^{\mathrm{n}}$, so $\mathrm{K}\left(\mathrm{g}^{\mathrm{n}}\right)$ is bounded by $\max \left(\mathrm{K}\left(\mathrm{f}^{\mathrm{n}}\right), \mathrm{K}(\phi)^{2}\right)$. Since $\mathrm{f}$ is quasirational, the dilatation of its iterates is uniformly bounded, so the same is true for $\mathrm{g}$. In case (b) we use the nonrecurrent assumption on $\mathrm{U}$ to check the uniform bound on $\mathrm{K}\left(\mathrm{g}^{\mathrm{n}}\right)$. 


\section{Remarks.}

(1) The degree of $f$ may be decreased by surgery.

(2) This type of construction was invented by Douady and Hubbard to realize a polynomial-like map as a polynomial [DH1].

\section{$\S$ 6. Dynamics on the Ideal Boundary}

We now turn to the action of $\mathrm{f}$ on the ideal boundary of a component $\mathrm{K}$ of its Julia set, and apply the gluing procedures introduced above to complete the proof of realization for $\operatorname{Mod}(\mathrm{K}, \mathrm{f})$ (Theorem 3.4; see 6.10 below).

Definition. We define three rigid models for proper self-maps of the unit disk $\Delta$ :

(a) the elliptic model $\mathrm{z} \rightarrow \mathrm{e}^{2 \pi \mathrm{i} \theta} \mathrm{z}$ where $\theta$ is irrational;

(b) the hyperbolic model $\mathrm{z} \rightarrow \mathrm{z}^{\mathrm{d}}$ for some $\mathrm{d}>1$; and

(c) the parabolic model $\mathrm{z} \rightarrow \mathrm{P}_{\mathrm{d}}(\mathrm{z})$ for $\mathrm{d}>1$, where

$$
\mathrm{P}_{\mathrm{d}}(\mathrm{z})=\frac{\mathrm{z}^{\mathrm{d}}+\mathrm{a}}{1+\mathrm{az}}, \quad \text { for } \mathrm{a}=(\mathrm{d}-1)(\mathrm{d}+1)
$$

is the unique map of degree $d$ with a single critical point at $z=0$ and an indifferent fixed point of multiplicity three at $\mathrm{z}=1$.

\section{Remarks.}

(1) Let B(z) be one of the above models, $\phi: \mathbf{S}^{1} \rightarrow \mathbf{S}^{1}$ a quasisymmetric automorphism of B ( $\phi \mathrm{B}=$ $\mathrm{B} \phi)$. Then $\phi$ is actually a rotation $\mathrm{z} \rightarrow \alpha \mathrm{z},|\alpha|=1$, and $\mathrm{B}(\alpha \mathrm{z})=\alpha \mathrm{B}(\mathrm{z})$ throughout the disk. It is for this reason we call these models rigid. For the elliptic model $\alpha$ can be arbitrary; for the hyperbolic model we must have $\alpha^{\mathrm{d}}=1$; and in the parabolic case $\alpha=1$ (the only automorphism is the identity.)

(2) One may demonstrate these assertions for models (a) and (b) by lifting to the universal cover of $\mathbf{S}^{\mathbf{1}}$; in fact the only topological (orientation preserving) automorphisms of (a) and (b) are the rotations just described. Thus a topological map commuting with $\mathrm{z}^{\mathrm{d}}$ is determined by its value on any one of the (d-1) fixed points.

(3) The parabolic model (c) is topologically conjugate to the hyperbolic model (b) of the same degree, so its topological automorphism are also determined by their value on a fixed point.

(4) There is no quasisymmetric conjugacy carrying the indifferent fixed point of $P_{d}$ to a repelling fixed point of any other map, since a repelling point expands small neighborhoods at a geometric rate and an indifferent point does not. Thus a quasisymmetric automorphism of $\mathrm{P}_{\mathrm{d}}$ must fix its indifferent fixed point on $\mathbf{S}^{1}$ (the remaining fixed points are repelling) and, since an automorphism is determined by the image of a fixed point, it must be the identity.

(5) Similarly, a topological conjugacy between $z^{d}$ and $P_{d}$ cannot be quasisymmetric. Thus different rigid models are not quasisymmetrically conjugate on $\mathbf{S}^{1}$.

Now let $\mathrm{f}(\mathrm{z})$ be a rational map, and let $\mathrm{K}$ be an infinite component of its Julia set $\mathrm{J}$ such that $f(K)=K$. Then $f^{-1}(K)$ consists of a finite union of components of $J$, one of which is $K$ itself. Thus $f$ gives rise to map $f: I(K) \rightarrow I(K)$ on the ideal boundary of $K$, as discussed in $§ 5$. Our first goal is to study the dynamics of $\mathrm{f}$ on the ideal boundary of $\mathrm{K}$.

\section{Theorem 6.1.}

(a) Every component of $\mathrm{I}(\mathrm{K})$ is preperiodic, and there are only finitely many periodic components.

(b) On each periodic component of the ideal boundary, the first return map is quasisymmetrically conjugate to one of the rigid models.

(c) All but finitely many components of $\hat{\mathbf{C}}-\mathrm{K}$ map to their images by conformal isomorphism. 
(d) If $\mathrm{C}$ is a component of $\mathrm{I}(\mathrm{K})$ which is not periodic, then there are quasisymmetric maps $\phi$ and $\psi$ carrying $\mathrm{C}$ and $\mathrm{f}(\mathrm{C})$ to $\mathbf{S}^{1}$ and conjugating $\mathrm{f}$ to $\mathrm{z} \rightarrow \mathrm{z}^{\mathrm{d}}$ for some d (possibly equal to 1 ).

The proof of (a,c and d) is fairly short; the proof of (b) occupies Propositions 6.2-6.8, which give a case-by-case study of the way in which the dynamics of $\mathrm{f}$ on $\mathrm{K}$ fits in with its global dynamics.

Proof of 6.1(c) and (d). Let $U$ be a component of $\hat{\mathbf{C}}-K$. Notice that $\mathrm{f}^{-1}(K)$ consists of finitely many components of $J$ (one of them $K$ itself). If $U$ meets neither $f^{-1}(K)$ nor contains any of the finitely many critical points of $\mathrm{f}$, then as claimed in (c), $\mathrm{f}$ carries $\mathrm{U}$ to another component of $\hat{\mathbf{C}}-\mathrm{K}$ by conformal isomorphism (since it is a proper local homeomorphism of a disk).

On the other hand, if $U$ is one of the finitely many components which contain a critical point or meet $\mathrm{f}^{-1}(\mathrm{~K})$, we can still find an annulus $\mathrm{U}^{\prime} \subset \mathrm{U}$ such that $\partial \mathrm{U} \subset \partial \mathrm{U}^{\prime}$ and $\mathrm{f}$ maps $\mathrm{U}^{\prime}$ to $\mathrm{f}\left(\mathrm{U}^{\prime}\right)$ by a covering. In the coordinates on ideal boundaries obtained by separately uniformizing $U^{\prime}$ and $f\left(U^{\prime}\right)$ as standard annuli of the form

$$
\{\mathrm{z}: \mathrm{r}<|\mathrm{z}|<1\}
$$

$\mathrm{f}$ is conjugate to $\mathrm{z} \rightarrow \mathrm{z}^{\mathrm{d}}$ for some $\mathrm{d}$; but these coordinates differ only real-analytically from the standard coordinates on $\mathrm{I}(\mathrm{K})$, establishing (d).

Proof of 6.1(a). Eventual periodicity and finiteness of the number of periodic cycles for the action of $f$ on $\mathrm{I}(\mathrm{K})$ is an immediate consequence of the following:

Proposition 6.2. Let $U$ be a component of $\hat{\mathbf{C}}-\mathrm{K}$. Then $\mathrm{I}(\mathrm{U})$ eventually maps to $\mathrm{I}(\mathrm{V})$ where $\mathrm{V}$ is either a Siegel disk for $\mathrm{f}$ or one of the finitely many components of $\hat{\mathbf{C}}-\mathrm{K}$ containing a critical point or meeting $\mathrm{f}^{-1}(\mathrm{~K})$.

Proof. If $\mathrm{f}^{\mathrm{n}}(\mathrm{I}(\mathrm{U}))$ never encounters a critical point or $\mathrm{f}^{-1}(\mathrm{~K})$, then as discussed above $\mathrm{f}^{\mathrm{n}}$ maps $\mathrm{U}$ by conformal isomorphism for all $\mathrm{n}$. Then $\mathrm{f}^{\mathrm{n}}(\mathrm{U})$ must eventually cycle, for otherwise $\mathrm{f}$ would have a wandering domain (ruled out by [Sul2]). Clearly $\left\langle\mathrm{f}^{\mathrm{n}}\right\rangle$ is a normal family on $\mathrm{U}$, so $\mathrm{U}$ eventually maps onto a stable region for $\mathrm{f}$ on which the first return map is a conformal isomorphism of a disk. Such a stable region must be a Siegel disk.

It remains only show the periodic components of $\mathrm{I}(\mathrm{K})$ behave up to quasisymmetric conjugacy like one of the rigid models.

Definition. A real-analytic endomorphism $\mathrm{h}: \mathbf{S}^{1} \rightarrow \mathbf{S}^{1}$ is expanding if there is a smooth metric $\rho(\theta) \mathrm{d} \theta$ with respect to which the derivate of $\mathrm{h}$ is everywhere $>1$. The model example is $\mathrm{z} \rightarrow \mathrm{z}^{\mathrm{d}}, \mathrm{d}>1$.

Proposition 6.3. Any expanding $\mathrm{C}^{1+\alpha}$ orientation-preserving endomorphism of $\mathbf{S}^{1}$ is quasisymmetrically conjugate to $\mathrm{z} \rightarrow \mathrm{z}^{\mathrm{d}}$ for some $\mathrm{d}>1$.

Proof. This is a special case of two general facts: expanding maps on compact manifolds are classified up to topological conjugacy by their action on the fundamental group [Shub], and topological conjugacies between expanding conformal dynamical systems are quasiconformal [Sul3].

For the remainder of this section, $\mathrm{U}$ will be a component of $\hat{\mathbf{C}}-\mathrm{K}$ such that $\mathrm{I}(\mathrm{U})$ is periodic. Replacing $\mathrm{f}$ by an iterate we will assume $\mathrm{I}(\mathrm{U})$ is fixed. As discussed above, there is an annulus $\mathrm{U}^{\prime} \subset \mathrm{U}$ on which $\mathrm{f}$ is a covering map to its image.

Proposition 6.4. If $U^{\prime}$ can be chosen so it is a proper subset of $f\left(U^{\prime}\right)$ then $f$ is quasisymmetrically conjugate on $\mathrm{I}(\mathrm{U})$ to $\mathrm{z} \rightarrow \mathrm{z}^{\mathrm{d}}$ for some $\mathrm{d}>1$. 
Proof. Uniformizing U, $\mathrm{f}$ becomes a map defined near the boundary of the unit disk which carries an annular neighborhood of $\mathbf{S}^{1}$ properly over itself. By Schwarz reflection we obtain a holomorphic covering F : A $\rightarrow$ B, where A and B are two annuli containing $\mathbf{S}^{1}$ and $\mathrm{A}$ is a proper subset of B. It follows that $\mathrm{F}$ is expanding on $\mathbf{S}^{1}$ relative to the Poincare' metric $\left.\rho(\mathrm{z})\right|_{\mathrm{dz}} \mid$ on B. (This argument is identical to the discussion of the external map in [DH1].)

Let $\Omega$ denote $\hat{\mathbf{C}}-\mathrm{J}$. The next Proposition reduces the proof of Theorem 6.1 to the case that $\partial \mathrm{U}$ is part of the boundary of a periodic component of $\Omega$.

Proposition 6.5. Either $\partial U$ is contained in $\partial V$ for some component $V$ of $\Omega \cap U$, or $f: I(U) \rightarrow I(U)$ is quasisymmetrically conjugate to $\mathrm{z} \rightarrow \mathrm{z}^{\mathrm{d}}$.

Remark. We will see in $\S 7$ that it is actually possible for $\mathrm{K}$ to be enclosed in a nest of components of $\mathrm{J}$, so that $\mathrm{K}$ (and hence $\partial \mathrm{U}$ ) does not meet the boundary of any component of $\Omega$.

Proof. Since $\mathrm{K}$ is a component of $\mathrm{J}$, there exist disjoint simple closed curves $\gamma_{\mathrm{n}}$ lying in $\mathrm{U} \cap \Omega$ such that $\gamma_{n}$ lies in an $r_{n}$ neighborhood of $\partial U$, where $r_{n} \rightarrow 0$ (apply Zoretti's theorem [W, p.109]). If $\partial \mathrm{U}$ is not contained in $\partial \mathrm{V}$ for some component $\mathrm{V}$ of $\Omega$, then these curves lie in infinitely many distinct components of $\Omega$.

Pick $\gamma_{n}$ sufficiently large that the annulus $V^{\prime}$ bounded by $\partial \mathrm{U}$ and $\gamma_{\mathrm{n}}$ contains no critical values of $\mathrm{f}$, and such that $\gamma_{\mathrm{n}}$ does not lie in a periodic component of $\Omega$ (this is possible because there are only finitely many periodic components.) Let $\mathrm{U}^{\prime}$ denote the (unique) component of $\mathrm{f}^{-1}\left(\mathrm{~V}^{\prime}\right)$ which lies in $\mathrm{U}$, such that $U^{\prime}$ is bounded by $\partial U$ and some pre-image of $\gamma_{n}$. Since $\gamma_{n}$ is disjoint from its pre-image (by virtue of lying in an aperiodic component), either $U^{\prime} \subset \mathrm{V}^{\prime}$ or $\mathrm{V}^{\prime} \subset \mathrm{U}^{\prime}$ (equality cannot hold). But the modulus of $U^{\prime}$ is less than or equal to that of $V^{\prime}$, so $U^{\prime}$ is a proper subset of $V^{\prime}=f\left(U^{\prime}\right)$. The preceding proposition applies to complete the proof.

We may now assume that $\partial \mathrm{U} \subset \partial \mathrm{V}$ for some component $\mathrm{V}$ of $\Omega \cap \mathrm{U}$. (We may not assume $\mathrm{U}=\mathrm{V}$ and indeed there may be regions in $U$ whose images cover the whole Riemann sphere.) Using the classification of stable regions [Sul3], the following three propositions complete the proof of Theorem 6.1 .

Proposition 6.6. If $\mathrm{V}$ is a Herman ring or a Siegel disk, then $\mathrm{f}: \mathrm{I}(\mathrm{U}) \rightarrow \mathrm{I}(\mathrm{U})$ is real-analytically (and hence quasisymmetrically) conjugate to an irrational rotation $\mathrm{z} \rightarrow \mathrm{e}^{\mathrm{i} \theta} \mathrm{z}$.

Proof. If $\mathrm{V}$ is a Siegel disk then $\mathrm{U}=\mathrm{V}$ and $\mathrm{f}$ is already conformally conjugate to an irrational rotation of the uniformization of $\mathrm{V}$. If $\mathrm{V}$ is a Herman ring then $\mathrm{f}$ is conformally conjugate to an irrational rotation of the standard annulus uniformizing $\mathrm{V}$, one of whose ideal boundary components differs from that of $\mathrm{U}$ only by a real-analytic transformation.

Proposition 6.7. If $\mathrm{V}$ is an attracting or superattracting basin, or a parabolic basin whose indifferent fixed point is not contained in $\partial \mathrm{U}$, then $\mathrm{f}: \mathrm{I}(\mathrm{U}) \rightarrow \mathrm{I}(\mathrm{U})$ is quasisymmetrically conjugate to $\mathrm{z} \rightarrow \mathrm{z}^{\mathrm{d}}$ for some $\mathrm{d}>1$.

Proof. Let $\mathrm{W} \subset \mathrm{V}$ be a Jordan domain such that $\mathrm{f}(\mathrm{W})$ is a proper subset of $\mathrm{W}$ and $\overline{\mathrm{W}}$ does not meet $\partial \mathrm{U}$. In the attracting and superattracting cases, we may take $\mathrm{W}$ to be a small round disk centered at the attracting fixed point in $\mathrm{V}$. In the parabolic case, take $\mathrm{W}$ to be a horoball-like region (a small petal or sepal of the 'Fatou flower') such that $\overline{\mathrm{W}}$ meets $\partial \mathrm{V}$ only at the indifferent fixed point.

Let $\mathrm{W}_{\mathrm{n}}$ denote the component of the nth pre-image of $\mathrm{W}$ which contains $\mathrm{W}$. Then

$$
\overline{\mathrm{W}_{1}} \subset \overline{\mathrm{W}_{2}} \subset \ldots \subset \mathrm{U} \quad \text { and }: \mathrm{W}_{\mathrm{n}}=\mathrm{V} \text {. }
$$


(In the parabolic case, since $\mathrm{f}(\partial \mathrm{U})=\partial \mathrm{U}$, the boundary of $\mathrm{U}$ avoids the grand orbit of the indifferent fixed point.)

For each $\mathrm{n}$, there is unique component of $\partial \mathrm{W}_{\mathrm{n}}$ which together with $\partial \mathrm{U}$ bounds an annular region $\mathrm{A}_{\mathrm{n}}$ disjoint from $\mathrm{W}_{\mathrm{n}}$ (Figure 6.1).

$A_{n}$ lies within an $r_{n}$ neighborhood of $\partial U$, where $r_{n} \rightarrow 0$; otherwise there is a continuum in $\partial V$ which lies in $\mathrm{U}$ and meets $\partial \mathrm{U}$, contradicting the assumption that $\mathrm{K}$ is a component of the Julia set.

Choosing $n$ large enough, we can insure that $A_{n}$ contains no critical values of $f$. Then $A_{n+1}$ is a component of $\mathrm{f}^{-1}\left(\mathrm{~A}_{\mathrm{n}}\right)$, as well as a proper subset of $A_{n}$, so Proposition 6.4 applies to complete the proof.

Proposition 6.8. If $\mathrm{V}$ is a parabolic basin, with indifferent fixed point contained in $\partial \mathrm{U}$, then $\mathrm{f:} \mathrm{I}(\mathrm{U}) \rightarrow \mathrm{I}(\mathrm{U})$ is quasisymmetrically conjugate to $\mathrm{z} \rightarrow \mathrm{P}_{\mathrm{d}}(\mathrm{z})$ for some $\mathrm{d}>1$, where $\mathrm{P}_{\mathrm{d}}$ is the parabolic rigid model.

Proof. We begin by uniformizing $\mathrm{U}$. Then $\mathrm{f}$ and $\mathrm{V}$ carry over to the unit disk $\Delta$, where we continue to denote them by $f$ and $V$. The map $f$ is is defined only on a subset of the disk, but this subset includes $V$ as well as an annular neighborhood of $\mathbf{S}^{1}$. By Schwarz reflection $\mathrm{f}$ is analytic on a neighborhood of $\mathbf{S}^{1}$, and has a unique indifferent fixed point on $\mathbf{S}^{1}$, corresponding to the indifferent fixed point in $\partial \mathrm{U}$. We may normalize so this fixed point is at $\mathrm{z}=1$.

Let $\mathrm{W} \subset \mathrm{V}$ be a small horoball touching the circle at the fixed point. For simplicity choose $\mathrm{W}$ such that its boundary avoids the grand orbits of the critical points. As before, we let $\mathrm{W}_{\mathrm{n}}$ denote the component of $\mathrm{f}^{-\mathrm{n}}(\mathrm{W})$ which contains $\mathrm{W}$. There is a unique component $\mathrm{F}_{\mathrm{n}}$ of $\partial \mathrm{W}_{\mathrm{n}}$ lying between $\partial \mathrm{U}$ and $\mathrm{W}_{\mathrm{n}}$, and meeting $\partial \mathrm{U}$ at the nth preimages of 1 . For $\mathrm{n}$ sufficiently large, $\mathrm{F}_{\mathrm{n}}$ lies in the annulus near the boundary of $\Delta$ on which $\mathrm{f}$ is a covering.

Carrying out the same discussion with $f$ replaced by $P_{d}$ (where $d=$ the degree of $f$ on $\mathbf{S}^{1}$ ), we obtain a similar picture in another copy of the unit disk. Construct a quasiconformal map $\phi$ carrying the 'fundamental domain' A bounded by $\mathrm{F}_{\mathrm{n}}$ and $\mathrm{F}_{\mathrm{n}+1}$ to the corresponding region for $\mathrm{P}_{\mathrm{d}}(\mathrm{z})$ (Figure 6.2). We can arrange that the map is Lipschitz and commutes with $f$ on the boundary of A.

Now use the dynamics on both sides to extend $\phi$ to a conjugacy between $f$ and $P_{d}$ on the pinched annulus bounded by $F_{n}$ and $\mathbf{S}^{1}$. The resulting map commutes with $f$ on this pinched region and has a quasiconformal extension to the full disk because of our Lipschitz requirement. Thus it induces a 
Figure 6.2. The parabolic case.

quasisymmetric conjugacy on $\mathbf{S}^{1}$, the ideal boundary of $\mathrm{U}$.

Proof of 6.1(b). Let I(U) be a periodic component of the ideal boundary of K. Apply Proposition 6.5 if $\partial \mathrm{U}$ is not contained in $\partial \mathrm{V}$ for $\mathrm{V}$ a component of $\Omega \cap \mathrm{U}$; otherwise $\mathrm{V}$ is a Siegel disk, Herman ring, or an attracting, superattracting or parabolic basin; apply $6.6,6.7$ or 6.8 as appropriate.

Using the preceding analysis of the dynamics on the ideal boundary of $\mathrm{K}$, we may now apply surgery to simplify the dynamics of $\mathrm{f}$ in the complement of $\mathrm{K}$ by pasting in rigid models.

Proposition 6.9. There is a stable conjugacy $(\mathrm{K}, \mathrm{f}) \sim(\mathrm{J}(\mathrm{g}), \mathrm{g})$, where $\mathrm{g}(\mathrm{z})$ is a rational map with the following properties. The Julia set $\mathrm{J}(\mathrm{g})$ is connected, and the stable regions of $\mathrm{g}$ are Siegel disks, superattracting and parabolic basins. On each stable region the first return is conformally conjugate to one of the three rigid models. If $\mathrm{c}$ is a critical point in $\hat{\mathbf{C}}-\mathrm{J}(\mathrm{g})$ which does not lie in a stable region, then there is a least $\mathrm{n}$ such that $\mathrm{g}^{\mathrm{n}}(\mathrm{c}) \varepsilon \mathrm{U}$ and $\mathrm{U}$ is a stable region; and

(a) if $\mathrm{U}$ is a Siegel disk, $\mathrm{g}^{\mathrm{n}}(\mathrm{c})$ coincides with the indifferent period cycle which forms the center of the disk;

(b) if $U$ is a superattracting basin, $g^{n}(c)$ lands on the superattracting periodic cycle; and

(c) if $\mathrm{U}$ is a parabolic basin, $\mathrm{g}^{\mathrm{n}+\mathrm{k}}$ (c) is also a critical point, where $\mathrm{k} \geq 0$ is the least integer such that $\mathrm{g}^{\mathrm{k}}(\mathrm{U})$ contains a critical point.

\section{Remarks.}

(1) It follows that the quotient Riemann surface $\mathrm{S}(\mathrm{g})$ consists of foliated disks and triplypunctured spheres. In particular it has no moduli.

(2) When there are parabolic regions, one must take care in constructing $g(z)$ since the critical point in the periodic basin cannot be made periodic. This is the reason for the convention in (c). Without such care it may not be true that every element of $\operatorname{Mod}(\mathrm{J}(\mathrm{g}), \mathrm{g})$ is isotopic to a Möbius transformation.

(3) The degree of $g$ is $>1$. This is implicit in our assumption that $\mathrm{K}$ (and hence $\mathrm{J}(\mathrm{g})$ ) is not a single point. 
Proof. We construct a quasirational map $\mathrm{q}(\mathrm{z})$ by applying the gluing lemma finitely many times to components of the complement of $\mathrm{K}$.

Consider first components $U$ such that $\mathrm{I}(\mathrm{U})$ is periodic (by 6.1(a), there are only a finite number of such $\mathrm{U})$. For simplicity assume that $\mathrm{I}(\mathrm{U})$ is actually fixed by $\mathrm{f}$. Using the part (a) of the Gluing Lemma, define $g(z)$ on $U$ by pasting in the rigid model to which $f$ is quasisymmetrical conjugate by Theorem 6.1(b).

Now work backwards along the pre-images of the periodic components of $\mathrm{I}(\mathrm{K})$, pasting in copies of $\mathrm{z} \rightarrow \mathrm{z}^{\mathrm{d}}$ using part (b) of the gluing lemma and 6.1(d). Whenever $\mathrm{d}>1$, choose the maps $\psi$ and $\phi$ such that under iteration the critical point lands on 0 in the standard coordinate systems for the rigid models. In other words, assure that the preperiodic critical points introduced land on the center of a Siegel disk in case (a), the superattracting periodic point in case (b), and the unique critical point in the immediate basin in case (c).

Once we have dealt with $\mathrm{f}^{-\mathrm{k}}$ of the periodic components of $\mathrm{I}(\mathrm{K})$ for $\mathrm{k}$ large enough, $\mathrm{f}$ maps all the remaining components of $\hat{\mathbf{C}}-\mathrm{K}$ by conformal isomorphism to their images. At this point we stop altering $f$.

The result is a quasirational map $q(\mathrm{z})$, agreeing with $\mathrm{f}$ on $\mathrm{K}$, such that $\mathrm{q}^{-1}(\mathrm{~K})=\mathrm{K}$. The map $\mathrm{q}(\mathrm{z})$ is quasiconformally conjugate to a rational map $\mathrm{g}(\mathrm{z})$, so we have a stable conjugacy $(\mathrm{K}, \mathrm{f}) \sim(\mathrm{F}, \mathrm{g})$, where $\mathrm{F}$ is a connected nowhere dense totally invariant set for $\mathrm{g}$.

We must show $\mathrm{F}=\mathrm{J}(\mathrm{g})$. It is enough to show the boundaries of the periodic components of $\hat{\mathbf{C}}-\mathrm{F}$ are in $\mathrm{J}(\mathrm{g})$, since their grand orbits are dense in $\mathrm{F}$. This is clear for the hyperbolic and parabolic models; for the elliptic case, we need only rule out the possibility that $\mathrm{g}(\mathrm{z})$ is an irrational rotation of the whole Riemann sphere, and $\mathrm{F}$ is an invariant circle. This could only arise if $\mathrm{K}$ is a quasicircle bounding a Siegel disk or Herman ring on both sides (as we have seen in 6.5-6.8). But then the iterates of $\mathrm{f}$ form a normal family on an annular neighborhood of $\mathrm{K}$, so $\mathrm{K}$ is not a component of the Julia set.

Since all automorphisms of the rigid models are essentialy conformal, we have good control over the stable automorphisms of $(\mathrm{J}(\mathrm{g}), \mathrm{g})$. This is made precise by the following proposition, which completes the proof of Theorem 3.4.

Proposition 6.10. The stable conjugacy $(\mathrm{K}, \mathrm{f}) \sim(\mathrm{J}(\mathrm{g}), \mathrm{g})$ establishes an isomorphism $\operatorname{Mod}(\mathrm{K}, \mathrm{f}) \simeq$ $\operatorname{Mod}(\mathrm{J}(\mathrm{g}), \mathrm{g})$. Every $[\phi]$ in the latter group has a unique representative $\phi$ which is conformal off the Julia set and commutes with $\mathrm{g}$ on the whole Riemann sphere. If $\mathrm{K}$ carries no invariant line field, $\phi$ is globally conformal and $\operatorname{Mod}(\mathrm{J}(\mathrm{g}), \mathrm{g}) \simeq \operatorname{Aut}(\mathrm{g})$.

Remark. Even without line field assumptions, the representative $\phi$ establishes an isomorphism $\operatorname{Mod}(\mathrm{J}(\mathrm{g}), \mathrm{g}) \simeq \operatorname{Mod}(\mathrm{g})$.

Proof. That the conjugacy gives an isomorphism is immediate from the definitions.

Let $[\phi]$ be an element of $\operatorname{Mod}(\mathrm{J}(\mathrm{g}), \mathrm{g})$. We claim the ideal boundary values of $\phi$ on any component of $\mathrm{I}(\mathrm{J}(\mathrm{g}))$ coincide with those of a conformal map commuting with g. If so, then by Cor. 5.4 there is an isotopy connecting $\phi$ to a unique quasiconformal map which is conformal on $\Omega=\hat{\mathbf{C}}-\mathrm{J}(\mathrm{g})$ and commutes with $\mathrm{g}$ everywhere. But if $\mathrm{J}(\mathrm{g})$ carries no line field, such a map is conformal, and hence $[\phi]$ is represented by an element of Aut(g), completing the proof.

To establish the claim, we begin by replacing $g$ with a suitable power so that all the periodic components of $\Omega$ are actually fixed. By construction, the fixed components of $\Omega$ are either Siegel disks, parabolic basins or superattracting basins, which are conjugate to rigid models after uniformization. On the ideal boundary of each fixed component, $\phi$ establishes a quasisymmetric conjugacy between two of the models.

As remarked earlier, the two models must be identical, and up to composition with uniformizing maps, $\phi$ is a rotation commuting with the dynamics throughout the disk. Thus the ideal boundary values 
of $\phi$ coincide with those of a conformal map commuting with $\mathrm{g}$.

Now let $\mathrm{U}$ be a preperiodic component of $\Omega$, and let $\mathrm{V}$ be $\phi(\mathrm{U})$. Then there is a least integer $\mathrm{k}$ such that $\mathrm{g}^{\mathrm{k}}(\mathrm{U})$ (and consequently $\mathrm{g}^{\mathrm{k}}(\mathrm{V})$ ) are components fixed by $\mathrm{g}$. If $\mathrm{g}^{\mathrm{k}}$ maps $\mathrm{U}$ by degree 1 , then the same is true for $\mathrm{V}$ and $\mathrm{g}^{-\mathrm{k}^{\circ}} \phi^{\circ} \mathrm{g}^{\mathrm{k}}$ extends to a conformal isomorphism from $\mathrm{U}$ to $\mathrm{V}$ with the same ideal boundary values of $\phi$ as claimed.

On the other hand, if $\mathrm{U}$ and $\mathrm{V}$ map by degree > 1, then by construction $\mathrm{g}^{\mathrm{k}}$ (when restricted to $\mathrm{U}$ or V) has a unique critical value, which either coincides with the center of a Siegel disk or the critical point in a parabolic or superattracting basin. Then the conformal extension of the boundary values of $\phi$ to a map from $\mathrm{g}^{\mathrm{k}}(\mathrm{U})$ to $\mathrm{g}^{\mathrm{k}}(\mathrm{V})$ carries the critical value to the critical value, and hence there is a lift $\mathrm{g}^{-} \mathrm{k}^{\circ} \phi^{\circ} \mathrm{g}^{\mathrm{k}}$ which is a conformal isomorphism between $\mathrm{U}$ to $\mathrm{V}$ and whose boundary values agree with those of $\phi$.

Thus on each component of $\Omega, \phi$ can be replaced by a conformal map commuting with $\mathrm{g}$.

\section{§ 7. Example: A Julia Set with a Buried Quasicircle.}

We will describe a Julia set with a component $K$ such that $f(K)=K$ but $K$ does not meet the boundary of any component of $\Omega$. We refer to such a $\mathrm{K}$ as a buried component. For comparison we recall some topological properties of Kleinian groups.

Proposition 7.1. Let $\Gamma$ be a finitely generated Kleinian group with limit set $\Lambda$ and domain of discontinuity $\Omega$. Then:

(a) The diameters of the components of $\Omega$ tend to zero.

(b) Any buried component of $\Lambda$ is a single point.

(c) Fixed points of elements of $\Gamma$ are dense in any component of $\Lambda$ which is not a point. (In particular any such component is the limit set of its stablilizer.)

(d) Under the action of $\Gamma$, the components of $\Lambda$ which are not points fall into finitely many equivalence classes.

Part (a) is proved by Maskit [Mskt]; parts (b-d) can be verified inductively using the decomposition theorem $[\mathrm{AM}]$. In [A] Abikoff gives an example of a limit set with a buried point (as in (b) above).

In our example the analogues of (a-d) fail to hold. As usual, J will denote the Julia set, $\Omega$ the domain of normality $\hat{\mathbf{C}}-\mathrm{J}$.

Proposition 7.2. Let $\mathrm{f}(\mathrm{z})=\mathrm{z}^{2}+\lambda \mathrm{z}^{3}$. For all $\lambda$ sufficiently small (but $\neq 0$ ),

(1) The diameters of the components of $\Omega$ do not tend to zero.

(2) The Julia set $\mathrm{J}$ is homeomorphic to [a Cantor set] $\mathrm{x} \mathrm{S}^{1}$.

(3) Every component of $\mathrm{J}$ is a quasicircle.

(4) J has uncountably many wandering components.

(5) J has uncountably many buried components.

(6) There is a unique buried component such that $f(K)=K$.

Remark. (6) shows the case dealt with by Proposition 6.5 can actually occur.

The Julia set for this map, with $\lambda=10^{-9}$, is rendered in the illustration at the beginning of this paper.

The above properties are easily verified from a picture of the dynamics of $\mathrm{f}$. When $\lambda$ is small, there is a large annulus A centered at zero, containing no critical values, and whose pre-image consists of an inner annulus I and an outer annulus $\mathrm{O}$, both nested inside of $\mathrm{A}$. O maps to A by degree 2 (by a small perturbation of $\mathrm{z} \rightarrow \mathrm{z}^{2}$ ), and I maps to $\mathrm{A}$ by degree 3 (by a small perturbation of $\mathrm{z} \rightarrow \lambda \mathrm{z}^{3}$ ) (Figure 7.1). The inner region enclosed by I maps to a neighborhood of infinity. Points not in A (such as 
Figure 7.1. An expanding map on nested annuli.

the critical values of $f$ ) tend to infinity under iteration. In particular $f$ is expanding.

Note that to construct this picture conformally, it is necessary that the annuli I and $\mathrm{O}$ fit inside of the large annulus $\mathrm{A}$; we need $\bmod (\mathrm{I})+\bmod (\mathrm{O}) \leq \bmod (\mathrm{A})$ (and an easy argument rules out equality if the picture occurs inside of a rational map). Since $I$ and $O$ are coverings of $A$ by degrees 2 and 3 , and $1 / 2+1 / 3<1$, the picture is possible. The map $\mathrm{z}^{\mathrm{n}}+\lambda \mathrm{z}^{\mathrm{m}}$ gives a similar example whenever $1 / \mathrm{n}+1 / \mathrm{m}<$ 1 ; however we do not know a simple example of a buried component for a rational map of degree less than 5 .

The Julia set can be constructed as $J=\cap f^{-n}(A)$. Using this construction, one arrives at a description of the topological dynamics. The components of the Julia set are circles nested between 0 from $\infty$. This gives an ordering to the components: $\mathrm{K}<\mathrm{L}$ if $\mathrm{K}$ separates $\mathrm{L}$ from 0 . The set of components is isomorphic to the one-sided shift on two symbols

$$
\Sigma_{2}=\{0,1\}^{\mathbf{N}}
$$

with the lexicographical ordering. With this identification, $\mathrm{f}$ determines a 2-to-1 endomorphism $\sigma$ of $\Sigma_{2}$, namely

$$
\begin{aligned}
\sigma\left(a_{1}, a_{2}, \cdots\right) & =\left(a_{2}, a_{3}, \ldots\right) & & \text { if } a_{1}=1, \\
& =\left(1-a_{2}, 1-a_{3}, \cdots\right) & & \text { if } a_{1}=0 .
\end{aligned}
$$

Thus $\mathrm{f}$ has exactly two fixed components, coded $(1,1,1,1 \ldots)$ and $(0,1,0,1,0, \ldots)$. The former is the outermost circle and the latter is the unique buried component $K$ such that $f(K)=K$.

The Julia set as a whole is homeomorphic to $\Sigma_{2} \times \mathbf{S}^{1}$. Identifying $\mathbf{S}^{1}$ with the unit circle in the complex plane, the topological dynamics is the skewed product

$$
\mathrm{F}\left(<\mathrm{a}_{\mathrm{i}}>, \mathrm{z}\right)=\left(\sigma<\mathrm{a}_{\mathrm{i}}>, \mathrm{z}^{5 \mathrm{a}_{1}-3}\right) .
$$

In other words $\mathrm{F}$ maps the circles in the outer annulus by degree 2 and those in the inner annulus by degree -3 . The expanding property implies the circles in $\mathbf{J}$ are actually quasicircles.

It is not hard to see how the surgery procedure of $\S 6$ applies to the dynamics of $f$ on the component $\mathrm{K}$. Since $\mathrm{K}$ is buried, one can find a small annulus enclosing $\mathrm{K}$ whose boundary consists of two other components of the Julia set. This annulus is mapped properly over itself, so $\mathrm{f}$ is expanding on the ideal boundary of $\mathrm{K}$. $\mathrm{K}$ is mapped to itself by degree -3 , and after pasting in rigid models we conclude that $(\mathrm{K}, \mathrm{f})$ is stably conjugate to $\left(\mathbf{S}^{1}, \mathrm{z}^{-3}\right)$. 


\section{$\S$ 8. Exposed Critical Points}

We conclude with the proof of a special case of the conjecture in $\S 3$. Perhaps the argument will shed light on the hard question of the existence of invariant line fields.

The following is a special case of a result of Strebel's [Str, Theorem 7].

Theorem 8.1. Let $U$ be a finitely connected domain in $\hat{\mathbf{C}}$, and let $\phi: \bar{U} \rightarrow \bar{U}$ be a quasiconformal homeomorphism which is the identity on $\partial \mathrm{U}$. Then there is a unique quasiconformal map $\psi$ of minimal dilatation among those isotopic to $\phi$ rel $\partial U$. The absolute value of the Beltrami differential $\mid \psi_{z}\left\langle\psi_{z}\right|$ is constant on $\mathrm{U}$ (in fact $\psi$ is a Teichmüller mapping of finite norm.)

Definition. We say $\mathrm{f}(\mathrm{z})$ has exposed critical points if every critical point lies in $\Omega$ or on the boundary of a component of $\Omega$.

Theorem 8.2. If $\mathrm{J}$ is connected and $\mathrm{f}$ has exposed critical points, then $\operatorname{Mod}(\mathrm{J}, \mathrm{f})$ is a finite group.

Proof. By Proposition 6.10, we may assume that every $[\phi]$ in $\operatorname{Mod}(\mathrm{J}, \mathrm{f})$ has a unique representative $\phi$ that commutes with $\mathrm{f}$ on the whole of $\hat{\mathbf{C}}$ and is conformal except possibly on J. (Altering $\mathrm{f}$ by stable conjugacy preserves the stable modular group.)

Let $\mathrm{E}$ be a finite union of components of $\Omega$ such that $\mathrm{E}^{-}$contains all the stable regions, all the critical values of $f$ and $E \subset f^{-1} E$. (E exists by assumption of exposed critical points.) It is easy to check that $\operatorname{Mod}(\mathrm{J}, \mathrm{f})$ has a subgroup of finite index consisting of $[\phi]$ such that $\phi$ is the identity on $\mathrm{E}$.

Let $\mathrm{V}=\hat{\mathbf{C}}-\mathrm{E}$. (If $\mathrm{V}$ is empty the proof is complete.) Each component of $\mathrm{V}$ is a finitely connected plane region. By Strebel's result, $\phi$ can be isotoped rel $\partial \mathrm{V}$ to a unique extremal map $\psi$ which is also the identity on E. Actually $\mathrm{V}$ has only finitely many multiply-connected components, so $\psi$ is the identity except on finitely many components of $\mathrm{V}$.

Now let $\mathrm{U}=\mathrm{f}^{-1}(\mathrm{~V})$. Then $\mathrm{f}: \mathrm{U} \rightarrow \mathrm{V}$ is a covering map. Since $\mathrm{f}^{\circ} \phi=\phi^{\circ} \mathrm{f}$ and $\psi$ is isotopic to $\phi$, we can form a sequence of lifted maps

$$
\psi_{0}=\psi, \quad \psi_{\mathrm{n}+1}=\mathrm{f}^{-1}{ }^{\circ} \psi_{\mathrm{n}}{ }^{\circ} \mathrm{f}
$$

where the lift is chosen so each $\psi_{\mathrm{n}}$ is again isotopic to $\phi$ and the identity on $\mathrm{E}$ (compare [M1, 8.1]).

By induction, $\psi_{\mathrm{n}}$ is conformal on $\mathrm{f}^{-\mathrm{n}}(\mathrm{E})$, and the maximal dilatation of $\psi_{\mathrm{n}}$ is the same as that of $\psi$ (since $\mathrm{f}$ is conformal). Consider the component $\mathrm{V}_{0}$ of $\mathrm{V}$ on which $\psi$ has maximal dilatation. For each $\mathrm{n}, \psi_{\mathrm{n}}$ has the same dilatation as $\psi$ and is isotopic to $\psi$ rel $\partial \mathrm{V}$, so by Strebel's uniqueness result $\psi=\psi_{\mathrm{n}}$ on $\mathrm{V}_{0}$. On the other hand, $\cup \mathrm{f}^{-\mathrm{n}}(\mathrm{E})=\Omega$ is an open dense set, so there is some $\mathrm{n}$ such that $\psi_{\mathrm{n}}$ is conformal on an open subset of $\mathrm{V}_{0}$. Since the dilatation of $\psi$ is constant on $\mathrm{V}_{0}$, we conclude that $\psi$ is conformal on the whole Riemann sphere (and the identity on E) hence $\psi=$ id.

Remark. The argument is a generalization of Thurston's theorem on the uniqueness of critically finite maps with given combinatorics [DH2]. There the uniqueness results for Teichmüller mappings of multiply punctured spheres play the same role as the more general result of Strebel's which we use above.

Acknowledgements. I'd like to thank Hubbard and Sullivan for instruction in rational maps, Marden for guidance in Kleinian groups, and the referee for helpful suggestions. 


\section{References}

[A] W. Abikoff. Some remarks on Kleinian groups. In Advances in the Theory of Riemann Surfaces, Annals of Math Studies 66 (1971), p.1-6.

[AM] W. Abikoff, B. Maskit. Geometric decompositions of Kleinian groups. Amer. J. Math. 99 (1977), p.687-698.

[AB] L. Ahlfors, L. Bers. Riemann's mapping theorem for variable metrics. Annals of Math. 72 (1960), pp. 385-404.

[BR] L. Bers, H. Royden. Holomorphic families of injections. To appear, Acta Mathematica.

[Bl] P. Blanchard. Complex analytic dynamics on the Riemann sphere. Bull. AMS 11 (1984), pp.85-141.

[DE] A. Douady, C. Earle. Conformally natural extensions of homemorphisms of the circle. Preprint.

[DH1] A. Douady, J. Hubbard. On the dynamics of polynomial-like mappings. Ann. sci. Ec. Norm. Sup. 18 (1985), pp.287-344.

[DH2] _ A proof of Thurston's topological characterization of rational maps. Preprint.

[H] M. Herman. Exemples de fractions rationelles ayant une orbite dense sur la sphere de Riemann. Bull. Soc.Math. de France 112 (1984), pp.93-142.

[J] K. Johansson. On the mapping class group of simple 3 manifolds. In Topology of Low Dimensional Manifolds, Springer-Verlag Lecture Notes 722 (1979), pp.48-66.

[Ker] S. Kerckhoff. The Nielsen realization problem. Annals of Mathematics 117 (1983), pp.235265.

[Kra] I. Kra. Deformation spaces. In A Crash Course on Kleinian Groups, Springer-Verlag Lecture Notes 400 (1974), pp.48-70.

[L] S. Lattes. Sur l'iteration des substitutions rationelles et les fonctions de Poincare'. CRAS Paris 166 (1918), pp.26-28.

[Ma] R. Mañe'. Instability of Herman rings. Inv. Math. 81 (1985), pp.459-472.

[MSS] R. Mañe', P. Sad, D. Sullivan. On the dynamics of rational maps. Ann. sci. Ec. Norm. Sup. t.16 (1983), pp.193-217.

[Mskt] B. Maskit. Intersection of component subgroups of Kleinian groups. In Discontinuous Groups and Riemann Surfaces, Annals of Math Studies 79 (1974), pp.349-367.

[Mc1] C. McMullen. Families of rational maps and iterative root-finding algorithms. To appear, Annals of Mathematics.

[Mc2] Braiding of the attractor and the failure of iterative algorithms. MSRI Preprint, 1986. 
[MB] J. Morgan, H. Bass (editors). The Smith Conjecture. Academic Press (1984).

[Shub] M. Shub. Expanding maps. In Global Analysis, AMS Proc. of Symp. XIV (1970), pp.273276.

[Str] K. Strebel. On quasiconformal mappings of open Riemann surfaces. Comm. Math. Helv. 53 (1978), pp.301-321.

[Sul1] D. Sullivan. Conformal dynamical systems. In Geometric Dynamics, Springer-Verlag Lecture Notes 1007 (1983), pp.725-752.

[Sul2] . Quasiconformal homeomorphisms and dynamics I: Solution of the Fatou-Julia problem on wandering domains. Annals of Math. 122 (1985), pp.401-418.

[Sul3] . Quasiconformal homeomorphisms and dynamics III: Topological conjugacy classes of analytic endomorphisms. Preprint.

[ST] D. Sullivan, W. Thurston. Extending holomorphic motions. To appear, Acta Mathematica.

[Th] W. Thurston. The Geometry and Topology of Three Manifolds. Lecture notes, Princeton University (1979).

[W] G. Whyburn. Analytic Topology. AMS Coll. Publ. 28 (1942).

[Z] H. Zieschang. Finite Groups of Mapping Classes of Surfaces. Springer-Verlag Lecture Notes 875 (1981).

Math Sciences Research Institute, Berkeley, CA 94720 